\title{
Wide-field compensation of monochromatic eye aberrations: expected performance and design trade-offs
}

\author{
Salvador Bará \\ Área de Óptica, Departamento Física Aplicada, Universidade de Santiago de Compostela, Galiza, Spain \\ Rafael Navarro \\ Instituto de Óptica “Daza de Valdés," Consejo Superior de Investigaciones Científicas, Madrid, Spain
}

Received April 7, 2002; revised manuscript received July 25, 2002; accepted August 13, 2002

\begin{abstract}
The optical quality of the human eye varies across the visual field. Hence an exact compensation of the eye aberration for a given field point can give rise to a less-than-optimum compensation in neighboring field regions. We have studied some aspects of this problem and present here an approach to design wide-field $\left(<10^{\circ}\right)$ optically thin correcting elements, e.g., phase plates, deformable mirrors, and liquid-crystal displays. Their expected performance is assessed using actual eye aberration data. Particular attention is given to the design of elements providing a minimum averaged $\mathrm{rms}$ residual aberration and those providing a nearly uniform rms residual aberration across a given field. (c) 2003 Optical Society of America

OCIS codes: $330.5370,330.4460,220.1000,080.3620$.
\end{abstract}

\section{INTRODUCTION}

In the past several years an intense research effort has been devoted to the measurement and improvement of the optical quality of the human eye. A detailed knowledge of the optical defects of individual eyes is of fundamental importance for applications in the fields of optometry, physiological optics, vision science, and clinical instrument design. It is well known that the eye, especially for large pupil diameters, behaves rather poorly as an imaging instrument because of the presence of optical aberrations, a fact pointed out by von Helmholtz a century and a half ago. ${ }^{1}$

The defects of the eye as an image-forming system can be quantitatively described by the wave aberration function, ${ }^{2}$ from which other useful metrics such as the point-spread function (PSF) or the modulation transfer function can be easily computed. The wave aberration function is conveniently expressed as a sum of polynomial terms, each one of them conveying information about a specific feature of the wave-front distortions. Among the several sets of mathematical functions particularly suited to this end, the well-known Zernike polynomials ${ }^{3-6}$ are of widespread use. Low-order eye defects such as the classical prismatic and spherocylinder refractive errors have for a long time been successfully measured and compensated for in clinical practice using relatively simple equipment. The measurement of higher-order aberrations and their successful compensation required the development of more sophisticated devices. In the last decade, a variety of aberrometers have been developed that allow determination of the precise shape of the eye's wave aberration with high spatial (and even temporal) resolution. Among others we can mention the HowlandHowland aberrometer, ${ }^{7}$ the Hartmann-Shack wave-front sensors, ${ }^{8-10}$ laser ray-tracing setups, ${ }^{11-13}$ and spatially resolved refractometers. ${ }^{14,15}$ The correction of these highorder refractive defects has also witnessed noticeable advances: Real-time aberration correction has been reported with adaptive optics systems using deformable mirrors ${ }^{16-18}$ or liquid-crystal devices, ${ }^{19,20}$ and compensation of static eye aberrations has been achieved using photoresist phase plates. ${ }^{21,22}$ New proposals for customized LASIK surgical procedures seek to compensate not only for the classical ametropies but for the high-order eye aberration terms.

Different aims feed the current trends for improving the optical quality of the eye in order to reach its diffraction limit. From the subject's viewpoint, diffractionlimited retinal images should help to achieve supernormal vision, with visual acuities nearly doubling the standard $20 / 20$ value. Although most subjects will never need such high acuity for everyday life, some specific vision-related tasks may benefit from this improvement. Research in physiological optics, especially in aspects related to the sampling and processing of retinal images, will also benefit from it. But perhaps the most conspicuous applications will be those related to the development of clinical instrumentation for high-resolution retinal imaging. Indeed the eye fundus images attainable using conventional imaging systems are severely blurred by monochromatic eye aberrations. Although such aberrations are not the only factor degrading retinal images (chromatic aberrations and scattering in ocular media also play an important role), correcting them will be an important step towards achieving high-resolution imagery for clinical diagnosis.

The optical quality of the eye varies strongly among subjects and for a given subject it varies noticeably across 
the field of view (FOV). This latter fact has been studied in detail using objective methods, ${ }^{23}$ suggesting that typical human eyes behave as wide-field imaging instruments whose optical quality is less than diffraction-limited but slowly varying across $\mathrm{a} \pm 10^{\circ}$-wide region about the foveal center. This optical quality decreases quickly for more eccentric field positions. It is worth noting that despite the approximately constant optical quality across the central field (measured by the rms wave aberration), the weight and importance of each individual Zernike term in the aberration function may vary rather strongly from one position to another. The slowly varying optical quality does not arise from the constancy of each individual aberration term but rather from a delicate balance of the contributions of the different Zernike terms. ${ }^{23}$ From this observation several questions naturally arise: If we correct exactly the optical aberrations for a given point in the visual field (say, for central foveal vision), how does it affect the image quality in the neighboring areas? To what extent can the optical quality of the eye be improved in certain regions of the visual field without worsening it noticeably in the remaining ones? The field-dependent decomposition of the aberration function in terms of individual Zernike polynomials suggests that no diffractionlimited correction can be achieved for extended FOV regions using a single thin optical element or surgical procedure. If so, what kind of trade-offs can be expected? How can we design an aberration-compensating element to achieve an optimum optical quality for a given field region in a particular eye?

Recent works ${ }^{24,25}$ have addressed the design of ophthalmic lenses to correct the eye's low-order peripheral re- fractive power errors (defocus and astigmatism) for wide visual fields. The design procedure involves the use of ray-tracing optimizing routines applied to model eyes to find the parameters of the surfaces of aspheric and astigmatic lenses which will provide a reasonable cancellation of the spherical and cylinder refractive error over the required field.

In this paper we present a different but complementary approach to the design of optical elements to compensate for the global aberration pattern of individual eyes across a wide field of view viz., $\sim \pm 10^{\circ}$ around the central fovea. This analytic method handles aberrations of any order and is not restricted to correction only of low-order refractive power errors. The analysis developed here is restricted to the design of optically thin elements that can be described by a transmittance function that in a first approximation does not depend on the visual field angle. As long as this angle is kept reasonably small, the assumption is reasonable for aberration-correcting elements such as photoresist phase plates manufactured on a flat substrate, deformable mirrors, and liquid-crystal displays. The results are of direct application to the design of wide-field elements to be used in high-resolution retinal imaging instruments. In addition, the results can be used as initial conditions for the nonlinear numerical optimization of the refractive surfaces of ophthalmic (contact) lenses drawn on curved, thick substrates.

The structure of this paper is as follows. In Section 2 we describe typical patterns of field-dependent eye aberrations taken from an earlier work. ${ }^{23}$ In Section 3 we introduce wide-field metrics of aberration correction and describe the optimization procedures for design of wide-field
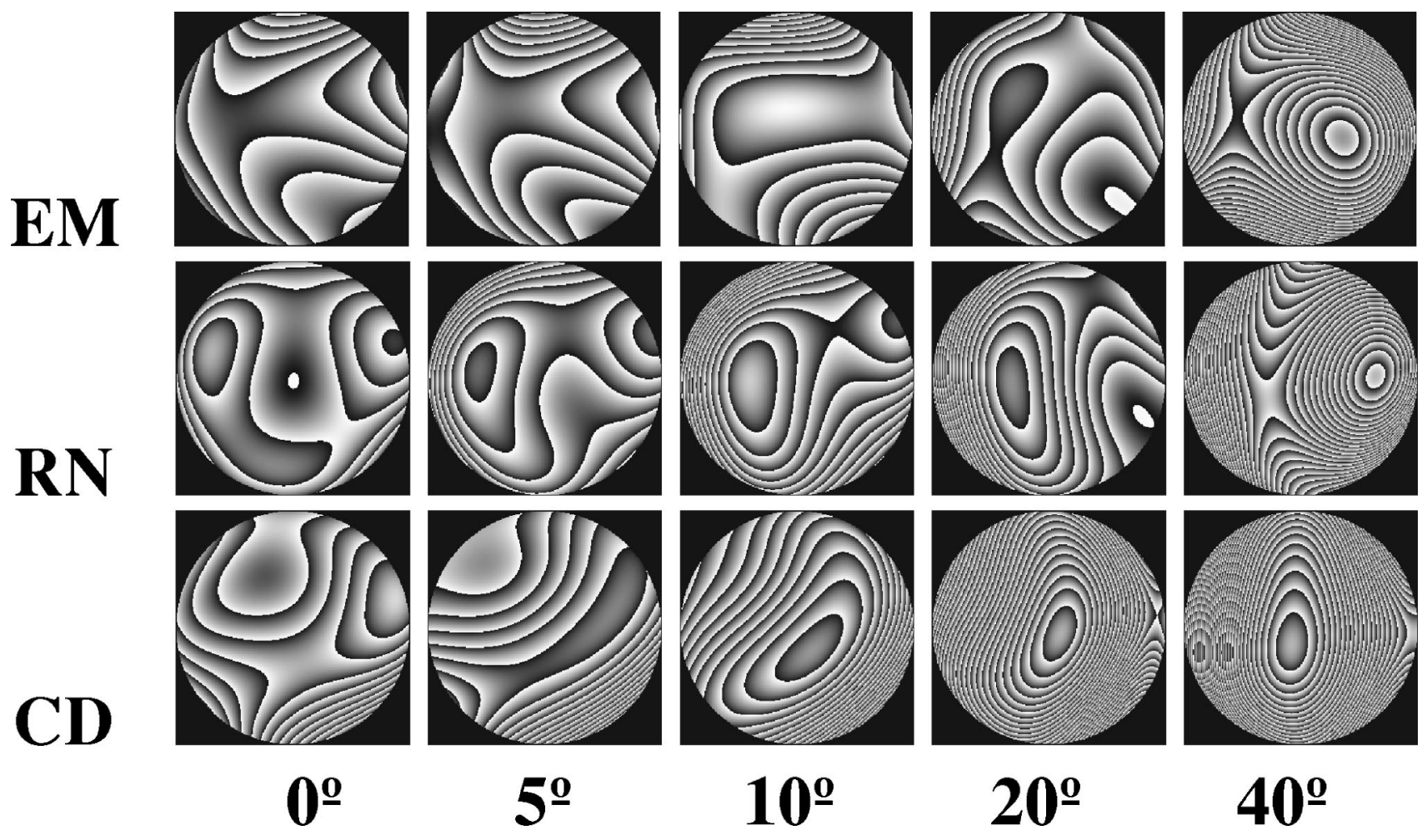

$20^{\mathbf{0}}$

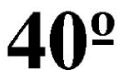

Fig. 1. Eye aberration maps for five different positions across the visual field of subjects EM, RN, and CD. Gray levels span one wavelength $(543 \mathrm{~nm})$. The on-axis defocus has been subtracted in all cases. 


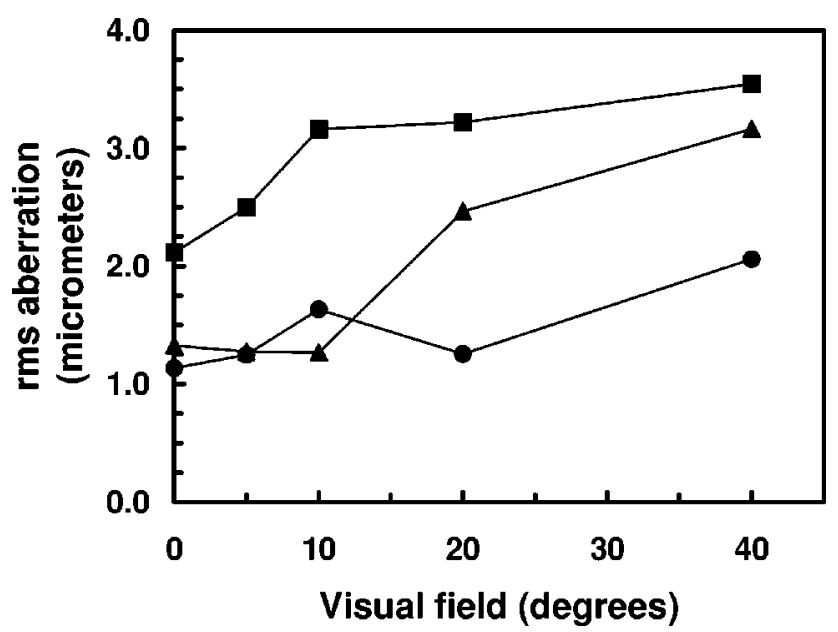

Fig. 2. Overall rms aberration including all terms (excepting tilts) of subjects EM (circles), RN (squares), and CD (triangles) for five positions across the visual field.

compensating elements adapted to individual eyes. Particular attention is given to the design of elements providing a minimum averaged residual $\mathrm{rms}$ aberration in a given field region and those providing a nearly uniform residual rms aberration across this FOV. In Section 4 these procedures will be applied to several sets of actual eye aberrations and their expected performance computed. Discussion, conclusions and future work are presented in Section 5.

\section{IMAGE QUALITY METRICS AND MONOCHROMATIC EYE ABERRATIONS ACROSS THE VISUAL FIELD}

To describe quantitatively the field dependence of the eye's aberrations, let us expand the wave aberration function $W_{e}(\mathbf{r}, \boldsymbol{\alpha})$ over the points $\mathbf{r}$ on the eye's exit pupil as a series of normalized Zernike polynomials with coefficients $a_{i}$ dependent on the angular position across the visual field $(\boldsymbol{\alpha})$. We have

$$
W_{e}(\mathbf{r}, \boldsymbol{\alpha})=\sum_{i=0}^{\infty} a_{i}(\boldsymbol{\alpha}) Z_{i}(\mathbf{r} / R),
$$

where $R$ is the radius of the pupil and $\boldsymbol{\alpha}$ is a twodimensional vector whose components are the angles formed by the chief ray corresponding to a given field position and the $x$ and $y$ axes defined on the eye's exit pupil. The Zernike polynomials $Z_{i}$ are labeled here by a single index $i$ embedding the two indices characterizing each term [radial $(n)$ and angular $(l)$ orders, respectively]. ${ }^{6}$ The first Zernike polynomial ( $i=0$ with $n=0$ ) is a constant term accounting for the average value of the aberration function and has no special relevance for image quality, so it is usually dropped from expansion (1). The next two terms with $i=1,2$ (both with $n=1$ ) correspond to global tilts in the wave fronts and hence describe prismatic effects. Terms with $i=3,4,5$ (all of them with $n=2)$ correspond to spherical ametropy $(l=0)$ and astigmatism $(l=2$ and $l=-2)$. Terms with $i>5$ ac- count for higher-order aberrations $(n>2)$ that have been shown to affect noticeably the image quality of the human eye. $8,16,26,27$

Following the recommendations in Ref. 6, here the Zernike polynomials $Z_{i}$ are normalized such that the overlapping integral,

$$
\frac{1}{\pi R^{2}} \int_{P} Z_{i}(\mathbf{r} / R) Z_{j}(\mathbf{r} / R) \mathrm{d}^{2} r,
$$

equals 1 for $i=j$ and is zero otherwise. In Eq. (2) $P$ denotes integration over the area of the eye pupil. A useful parameter describing the overall magnitude of aberrations is the wave-front variance, defined for each field position as

$$
\sigma^{2}(\boldsymbol{\alpha})=\frac{1}{A_{p}} \int_{P}\left[W_{e}(\mathbf{r}, \boldsymbol{\alpha})-\bar{W}_{e}(\mathbf{r}, \boldsymbol{\alpha})\right]^{2} \mathrm{~d}^{2} r
$$

where as before $P$ denotes integration over the eye exit pupil, $A_{p}$ is the pupil area, and $\bar{W}_{e}(\mathbf{r}, \alpha)$ is the average value of the wave aberration over the pupil. One advantage of using the Zernike polynomial expansion on a circular pupil is that $\bar{W}_{e}(\mathbf{r}, \boldsymbol{\alpha})=a_{0}$. An even more useful property is that the wave-front variance can be written in terms of the normalized aberration coefficients $a_{i}$ in the compact form

$$
\sigma^{2}=\sum_{i=1}^{\infty} a_{i}{ }^{2} .
$$

Note that since the wave aberration depends on the visual angle $\boldsymbol{\alpha}$, the PSF and the modulation transfer function, as well as the wave-front variance, Strehl ratio, and other image quality metrics, will also depend on $\boldsymbol{\alpha}$.

Figure 1 illustrates the strong dependence of the overall wave aberration $W_{e}(\mathbf{r}, \boldsymbol{\alpha})$ on visual angle $\boldsymbol{\alpha} . \quad W_{e}(\mathbf{r}, \boldsymbol{\alpha})$ is plotted for three subjects (EM, RN, and CD) and five temporal field positions $\left(\alpha=0^{\circ}, 5^{\circ}, 10^{\circ}, 20^{\circ}\right.$ and $\left.40^{\circ}\right)$. These data were obtained using the laser ray tracing method. ${ }^{23}$ In these wrapped gray level plots, the gray

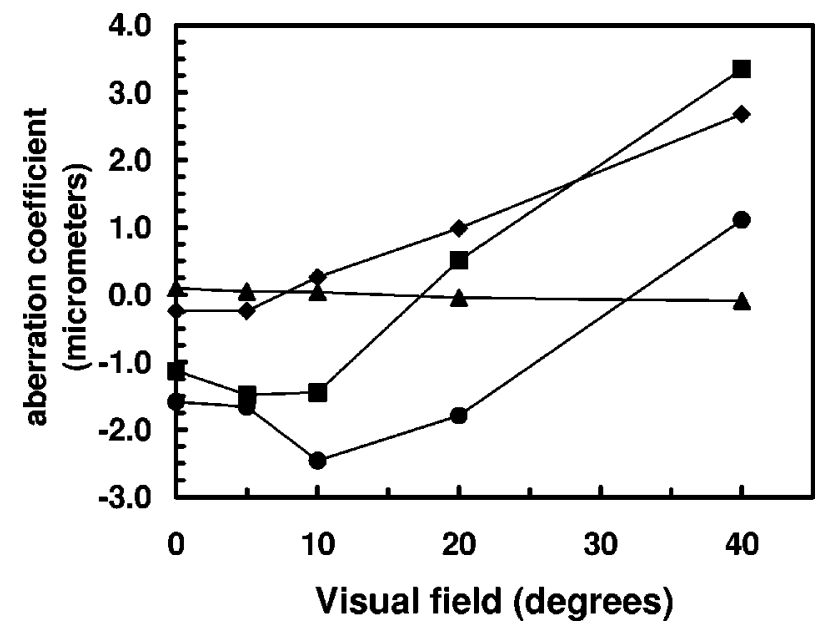

Fig. 3. Aberration coefficients $a_{i}$ for subject EM for five positions across the visual field. Circles, $i=4$ (defocus, including the on-axis measured value); squares, $i=5$ (astigmatism at $0^{\circ}$ or $90^{\circ}$ ); diamonds, $i=8$ (third-order coma along $x$ axis); and triangles, $i=12$ (spherical aberration). 
level range corresponds to one wavelength $(543 \mathrm{~nm})$ of optical path length. To help in visualizing the differences, the on-axis defocus term of each subject $a_{4}\left(0^{\circ}\right)$ (which can be easily compensated for using conventional ophthalmic lenses) has been subtracted from $W_{e}(\mathbf{r}, \boldsymbol{\alpha})$ in all cases. The associated rms wave aberration $\sigma(\boldsymbol{\alpha})$ (including defocus but not tilts) is plotted in Fig. 2 versus field angle $\boldsymbol{\alpha}$.

Individual Zernike aberration terms show a great variability across the visual field, even in the central region. As an example, Fig. 3 shows the aberration coefficients corresponding to $i=4$ (defocus), 5 (astigmatism at $0^{\circ}$ or $90^{\circ}$ ), 8 (third-order coma along the $x$ axis), and 12 (spherical aberration) for subject EM. The resulting lines are often far from being constant and may even suffer changes of sign. The general trend is that their absolute value tends to increase toward higher values of eccentricity.

\section{WIDE-FIELD COMPENSATION OF EYE ABERRATIONS}

As mentioned above, several technologies can be used to compensate for human eye aberrations beyond the conventional spherocylindrical ametropies. These compensation approaches rely on the use of a special purpose optical element-either static or dynamic-designed to imprint on the incoming wave fronts an aberration function of the same magnitude but reversed sign from that of the eye. Customized LASIK surgery seeks to obtain similar results, the main difference being that in the LASIK approach no additional optical elements are included in the optical path, but the cornea is reshaped to compensate for the optical defects.

Many of these compensating elements can be considered optically thin so that their effects can be satisfactorily described by a transmittance function $\exp \left[i k W_{c}(\mathbf{r})\right]$ that, for small enough visual fields, is independent of the field angle $\boldsymbol{\alpha}$. In other words the effect of a thin compensating element is essentially to introduce a local phase retardation $k W_{c}(\mathbf{r})$ on the incoming waves whose value depends on the pupil point being considered (r) but not on the incidence angle, as long as this is kept reasonably small (say, $\boldsymbol{\alpha} \leqslant 10^{\circ}$ ). When the compensating element is placed on a plane conjugated to the eye's pupil (or at a small axial distance from it: For typical human eye aberrations, small axial misalignments-up to a few millimeters-have only a minor effect on the compensation quality), ${ }^{28}$ the resulting residual wave aberration of the eye $W_{r}(\mathbf{r}, \boldsymbol{\alpha})$ can be described as the sum of the original eye aberration $W_{e}$ and the wave-front distortion $W_{c}$ introduced by the correcting element in the form

$$
W_{r}(\mathbf{r}, \boldsymbol{\alpha})=W_{C}(\mathbf{r})+W_{e}(\mathbf{r}, \boldsymbol{\alpha}) .
$$

From Eq. (5) it follows that for any single field position $\boldsymbol{\alpha}_{0}$, the residual eye aberration may in principle be perfectly canceled using a correcting element with $W_{c}(\mathbf{r})$ $=-W_{e}\left(\mathbf{r}, \boldsymbol{\alpha}_{0}\right)$. However, such a perfect cancellation cannot be simultaneously achieved for a set of points across the visual field. This effect can be seen in Fig. 4, whose second row shows the residual eye aberration of subject EM for two other field positions once the on-axis aberration $\left(\boldsymbol{\alpha}=0^{\circ}\right)$ has been completely cancelled
$\left[W_{r}(\mathbf{r}, \boldsymbol{\alpha})=W_{e}(\mathbf{r}, \boldsymbol{\alpha})-W_{e}\left(\mathbf{r}, 0^{\circ}\right)\right]$. The corresponding PSFs, numerically evaluated from $W_{r}(\mathbf{r}, \boldsymbol{\alpha})$, are displayed in the second row of Fig. 5. Note that for $\boldsymbol{\alpha}$ $=0^{\circ}$ the residual aberration is zero (flat gray level), giving rise to a diffraction-limited PSF (an Airy disk). However, the optical quality for off-axis points along the temporal field, nasal retina meridian $\left(\boldsymbol{\alpha}_{x}=5^{\circ}, 10^{\circ} ; \boldsymbol{\alpha}_{y}\right.$ $\left.=0^{\circ}\right)$, varies noticeably and is still far from its diffraction limit despite the overall improvement achieved with respect to the fully uncompensated case.

To develop methods for optimizing the compensating element, it is useful to expand $W_{c}(\mathbf{r})$ also in terms of normalized Zernike polynomials as
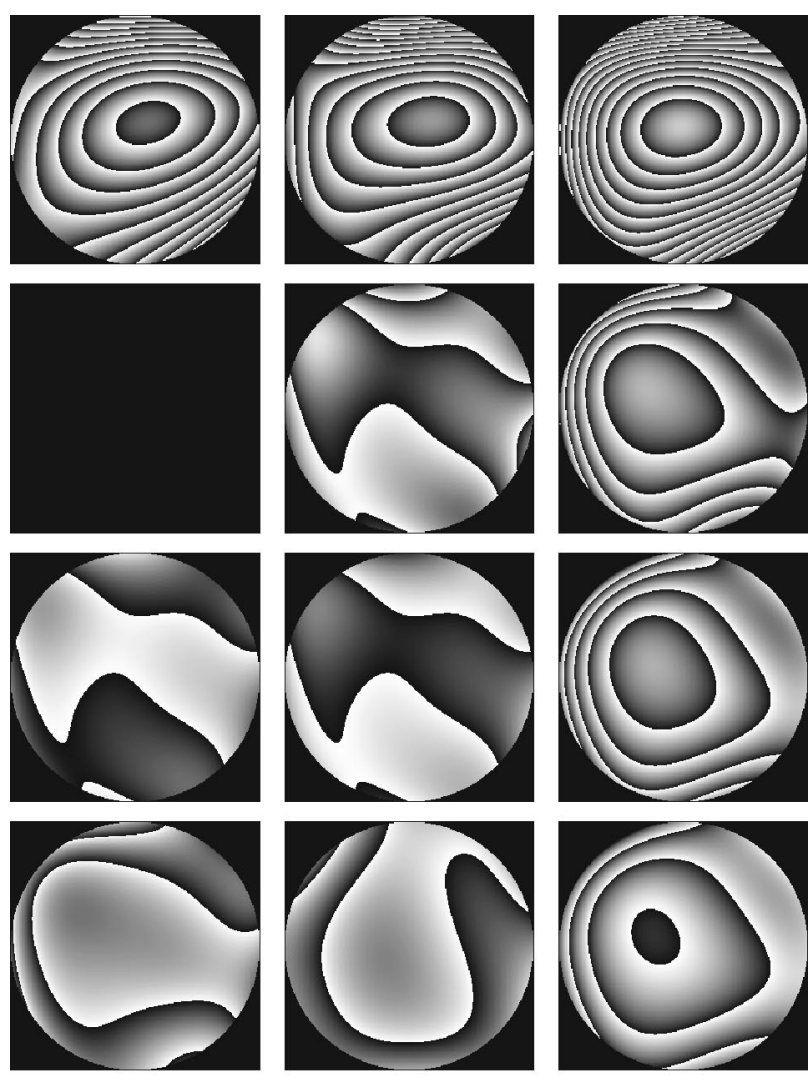

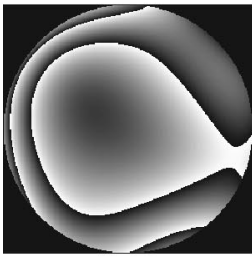

$\mathbf{0}^{\mathrm{o}}$

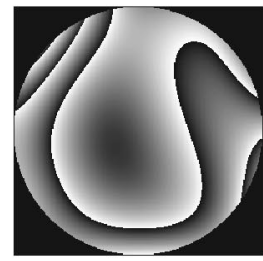

5은

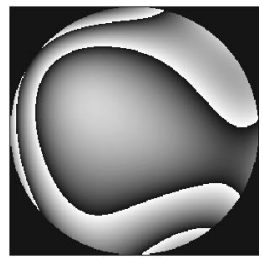

$10^{\mathbf{0}}$
Fig. 4. Maps of the EM wave aberration, with the gray-scale levels spanning one wavelength of optical path $(543 \mathrm{~nm})$. Pupil diameter $6.7 \mathrm{~mm}$. Each column corresponds to a different position within the foveal and parafoveal field: $0^{\circ}, 5^{\circ}$, and $10^{\circ}$, as noted. All terms in the aberration function have been included, excepting for the tilt. Upper row, uncompensated eye; second row, eye compensated with $w=[100]$; third row, same with $w=(1 / 2)[110]$; fourth row, same with $w=(1 / 3)$ [111]; fifth row, same with an optimal element designed to achieve the smallest uniform variance at these three positions of the field. Note the smaller residual aberration at $10^{\circ}$ and the slightly higher one at $0^{\circ}$ and $5^{\circ}$ in comparison with the fourth row. 

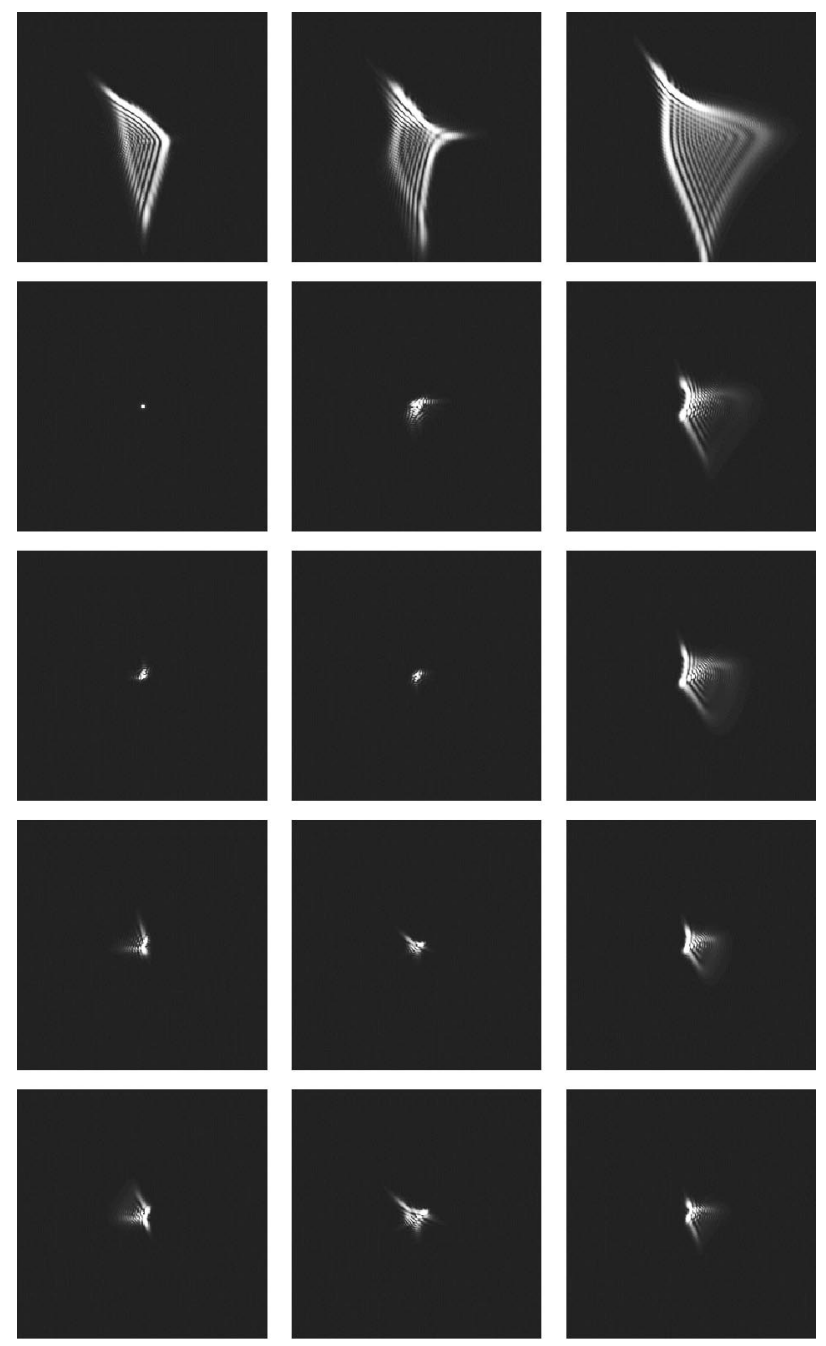

(0)

$$
\sigma^{2}(\boldsymbol{\alpha})=\sum_{i=1}^{N}\left[c_{i}+a_{i}(\boldsymbol{\alpha})\right]^{2}+\sum_{i=N+1}^{\infty}\left[a_{i}(\boldsymbol{\alpha})\right]^{2},
$$

where the first summation represents the residual aberration variance for the compensated terms while the second term is the contribution of the high-order uncompensated ones. This latter summation will always be present, since $N$ is finite; but taking into account that the magnitude of the individual eye aberrations decreases for high values of $i{ }^{29}$ the contribution of this sum can be made negligibly small (in principle) by taking $N$ high enough.

In Subsections 3.A and 3.B we will present analytical approaches for computing the optimum value of the set of coefficients $\left\{c_{i}\right\}$ necessary to achieve specific compensation goals. The first approach will seek to achieve the minimum averaged residual rms aberration in a given field of view with an arbitrary weighting of each field position. The second will look for solutions necessary to obtain a uniform residual $\mathrm{rms}$ aberration in a given number $M$ of points across the field of view. The number of solutions to this problem depends on the relative size of $M$ and $N$. For the most usual case $(M<N)$ there is an infinity of solutions differing in the amount of residual aberration left uncorrected. The uniform optical quality solution providing at the same time the minimum residual aberration compatible with it will also be derived.

\section{A. Minimum Residual Variance}

Computing the optimal values for the coefficients $c_{i}$ of a wide-field correcting element requires us to minimize a cost function according to the design goal. A common and useful approach is to choose as cost function a weighted average of the residual aberration variance $\sigma^{2}(\boldsymbol{\alpha})$ across the field of view; that is,

$$
\sigma^{2} \equiv\left\langle\sigma^{2}(\boldsymbol{\alpha})\right\rangle=\int_{A} \omega(\boldsymbol{\alpha}) \sigma^{2}(\boldsymbol{\alpha}) \mathrm{d}^{2} \boldsymbol{\alpha},
$$

where $\omega(\boldsymbol{\alpha})$ is a weighting function accounting for the relative importance assigned to each position over the field, normalized such that

$$
\int_{A} \omega(\boldsymbol{\alpha}) \mathrm{d}^{2} \boldsymbol{\alpha}=1
$$

where the sum has a finite limit of terms $(N)$ since any practical aberrometer gives information only about a finite, although possibly large, number of individual aberrations, and the compensating elements are fabricated according to these specifications. From Eqs. (1), (5), and (6) we can rewrite the residual aberration resulting from compensation in the convenient form

$$
W_{r}(\mathbf{r}, \boldsymbol{\alpha})=\sum_{i=1}^{\infty}\left[c_{i}+a_{i}(\boldsymbol{\alpha})\right] Z_{i}(\mathbf{r} / R),
$$

where the sum extends formally to infinity, with the proviso that $c_{i}=0$ for $i>N$, since modes of order higher than $N$ will not be included in the compensating element.

Taking advantage of the properties of the normalized Zernike polynomials, the residual aberration variance after compensation is then given by the simple expression
The bracketed term in (9) is simply a convenient shorthand notation for the weighted average operation. The residual wavefront variance at each field position is a useful measure of the optical quality of the compensated eye at that field point, and gives information about the expected loss of image quality particularly when the residual aberrations are small. Thus by minimizing $\sigma^{2}$ we get the best averaged optical quality in a given field region, taking into account the relative importance assigned to each point through the arbitrary choice of the function $\omega(\boldsymbol{\alpha})$.

Substituting Eq. (8) into Eq. (9) and setting to zero the partial derivatives of $\sigma^{2}$ with respect to the coefficients of the correcting element it is straightforward to obtain the set of $c_{i}$ giving the smallest value for $\sigma^{2}$. They are 


$$
c_{i}=-\int_{\mathrm{A}} \omega(\boldsymbol{\alpha}) a_{i}(\boldsymbol{\alpha}) \mathrm{d}^{2} \boldsymbol{\alpha}, \quad(i=1, \ldots, N) .
$$

Each Zernike coefficient of this compensating element is simply the weighted field average of the corresponding coefficient of the aberrated eye, with reversed sign. In shorthand notation this is equivalently written as

$$
c_{i}=-\left\langle a_{i}(\boldsymbol{\alpha})\right\rangle, \quad(i=1, \ldots, N) .
$$

Equations (9)-(11) are written on the assumption that the eye aberration is known for the continuum of values of $\boldsymbol{\alpha}$ within the region of interest. However in most cases the eye aberration will be measured at only a finite-and usually small—set of $M$ visual field positions $\left(\boldsymbol{\alpha}_{1}, \ldots, \boldsymbol{\alpha}_{M}\right)$ at which the cost function will be evaluated. In this case the computation of the optimum coefficients from these available data is the same as before, except we replace the continuous function $\omega(\boldsymbol{\alpha})$ by a weighted discrete sum of Dirac delta distributions centered at the measurement points in the form

$$
\omega(\boldsymbol{\alpha})=\sum_{k=1}^{M} \omega_{k} \delta\left(\boldsymbol{\alpha}-\boldsymbol{\alpha}_{k}\right),
$$

with the weights vector $\boldsymbol{\omega}=\left(\omega_{1}, \ldots, \omega_{M}\right)$ normalized as

$$
\sum_{k=1}^{M} \boldsymbol{\omega}_{k}=1
$$

so that the cost function becomes

$$
\sigma^{2}=\sum_{k=1}^{M} \omega_{k} \sigma^{2}\left(\boldsymbol{\alpha}_{k}\right)
$$

Consequently the optimal coefficients are given by

$$
c_{i}=-\sum_{k=1}^{M} \omega_{k} a_{i}\left(\boldsymbol{\alpha}_{k}\right) \text {. }
$$

Equation (16) is a discrete version of Eq. (11), and can be rewritten as Eq. (12), the discrete character of the weighted average operation being understood. Once the optimal coefficients have been calculated, the residual aberration variance of the compensated eye can be computed at any field position by substituting either Eq. (11) or Eq. (16) into Eq. (8).

\section{B. Uniform Residual Variance}

The solution given in the preceding paragraph allows us to achieve the smallest averaged residual aberration variance across a given field region, but it is worth noting that this does not guarantee that the residual variance of the compensated eye will be uniform (or the most uniform possible) at all points of the field. Some applications, especially those dealing with medium- to high-resolution retinal imaging in extended fields and including those using deconvolution techniques, may benefit from a uniform or nearly uniform optical quality, rather than from one minimum averaged but variable across the field. The price to pay for uniformity is that in this case the averaged residual aberration will usually be greater than in the minimum averaged approach. This does not necessarily mean that the resulting optical quality will be worse at all field points for the uniform approach than for the minimum averaged one. In fact, it may be even better in a significant region of the FOV.

The computation of the set of coefficients $c_{i}$ to achieve the most uniform residual aberration variance after compensation can be done in two equivalent ways. One of them is to minimize the variance of the $\sigma^{2}(\boldsymbol{\alpha})$ values, that is, to minimize the average $\eta$ of their squared differences with respect to their mean value in the region of interest of the visual field, or

$$
\eta \equiv\left\langle\left[\sigma^{2}(\boldsymbol{\alpha})-\left\langle\sigma^{2}(\boldsymbol{\alpha})\right\rangle\right]^{2}\right\rangle,
$$

where the brackets denote the weighted average operation on $\boldsymbol{\alpha}$. In this case the weighting function will be constant and given by $\omega(\boldsymbol{\alpha})=1 / A_{p}\left(A_{p}=\right.$ pupil area $)$ for all $\boldsymbol{\alpha}$ in the continuous case, and $\omega_{k}=1 / M(M=$ number of measurement points) for all $\boldsymbol{\alpha}_{\kappa}$ in the discrete one. The minimization is done by substituting for $\sigma^{2}(\boldsymbol{\alpha})$ in Eq. (17) its value from Eq. (8), setting to zero the partial derivatives of $\eta$ with respect to $c_{i}(i=1, \ldots, N)$, and solving for the coefficients $c_{i}$. This approach is straightforward although the notation is a bit cumbersome and will not be explicitly developed in this paper.

Here we have adopted an equivalent way that allows us to simplify somewhat the notation for the most practically relevant discrete case. After substituting $\sigma^{2}(\boldsymbol{\alpha})$ from Eq. (8) for $\left(\boldsymbol{\alpha}_{1}, \ldots, \boldsymbol{\alpha}_{M}\right)$-assuming that the contribution of the uncorrected modes $(i>N)$ is negligible and writing explicitly the averaging operation over the $M$ field positions-we get for $\eta$

$$
\eta=\frac{4}{M} \sum_{k=1}^{M}\left[\sum_{i=1}^{N}\left(A_{k i} c_{i}-\delta_{k i}\right)\right]^{2},
$$

where

$$
\begin{aligned}
A_{k i} & =a_{i}\left(\boldsymbol{\alpha}_{k}\right)-\left\langle a_{i}\left(\boldsymbol{\alpha}_{k}\right)\right\rangle, \\
\delta_{k i} & =(-1 / 2)\left[a_{i}{ }^{2}\left(\boldsymbol{\alpha}_{k}\right)-\left\langle a_{i}{ }^{2}\left(\boldsymbol{\alpha}_{k}\right)\right\rangle\right] .
\end{aligned}
$$

Now $\eta$ can be realized as $4 / \mathrm{M}$ times the square of the Euclidean norm of an $M$-dimensional vector $\boldsymbol{\nu}$ defined by

$$
\boldsymbol{\nu}=\mathbf{A c}-\mathbf{d},
$$

where $\mathbf{A}$ is an $M \times N$ matrix whose elements are $A_{k i}$, c is the column vector $\left\{c_{i}\right\}$ of size $(N \times 1)$ of Zernike coefficients of the correcting element, and $\mathbf{d}$ is a column vector of size $M \times 1$ whose elements $d_{k}(k=1, \ldots, M)$ are given by

$$
d_{k}=\sum_{i=1}^{N} d_{k i}
$$

Minimizing $\eta$ is thus equivalent to finding the vector $\mathbf{c}$ minimizing $\|\mathbf{A c}-\mathbf{d}\|$ for a given pair $\mathbf{A}$ and $\mathbf{d}$, which depend only on the individual eye aberration data [Eq. (19)].

The solutions for this kind of linear minimization problem are well known. Note that usually $M \neq N$ (i.e., the number $M$ of field points where the eye aberration is known and the number $N$ of modes included in the expansion of the aberration function of the correcting element do not have to be the same), so that $\|\mathbf{A c}-\mathbf{d}\|$ cannot be directly minimized for $c$ by inverting the matrix $\mathbf{A}$ to get 
$\mathbf{c}=\mathbf{A}^{-1} \mathbf{d}$ since $\mathbf{A}$ is nonsquare. Note also that even in the case of having $M=N$, and thus a square $\mathbf{A}$ matrix, $\mathbf{A}$ is singular. Indeed the rank of $\mathbf{A}$ equals, in the best case, the minimum of $(N, M-1)$. Each column of A corresponds to a single Zernike term and the elements of the column are the deviations between a set of data (the corresponding Zernike coefficient evaluated at a set of field points) and its average value, so that the sum along columns is strictly zero.

For $M>N$ (i.e., more data than unknowns) and rank (A) $=N$, the (unique) c which minimizes the Euclidean norm $\|\mathbf{A c}-\mathbf{d}\|$ can be easily computed by the standard least-squares solution ${ }^{30}$ as

$$
\mathbf{c}=\left(\mathbf{A}^{\mathrm{T}} \mathbf{A}\right)^{-1} \mathbf{A}^{\mathrm{T}} \mathbf{d},
$$

where $\mathbf{A}^{\mathrm{T}} \mathbf{A}$ (the superscript T stands for "transpose") is a square and nonsingular matrix so that its inverse exists.

For all other cases, including the most usual one in eye aberration compensation $(M<N)$, whatever the rank of A may be, the least-squares solution c minimizing $\|\mathbf{A c}-\mathbf{d}\|$ is no longer unique but there is an infinite set of solutions given $\mathrm{by}^{30}$

$$
\mathbf{c}=\mathbf{A}^{+} \mathbf{d}+\left(\mathbf{I}-\mathbf{A}^{+} \mathbf{A}\right) \mathbf{y},
$$

where $\mathbf{I}$ is the $N \times N$ identity matrix, $\mathbf{y}$ is any arbitrary column vector of size $N \times 1$, and $\mathbf{A}^{+}$stands for the generalized inverse of $\mathbf{A}$, or pseudoinverse, ${ }^{30,31}$ a matrix which can be defined through the Moore-Penrose conditions as

$$
\begin{aligned}
& \mathbf{A} \mathbf{A}^{+} \mathbf{A}=\mathbf{A}, \quad \mathbf{A}^{+} \mathbf{A} \mathbf{A}^{+}=\mathbf{A}^{+}, \\
& \left(\mathbf{A} \mathbf{A}^{+}\right)^{T}=\mathbf{A} \mathbf{A}^{+}, \quad\left(\mathbf{A}^{+} \mathbf{A}\right)^{T}=\mathbf{A}^{+} \mathbf{A} .
\end{aligned}
$$

Pseudoinverse computation through singular value decomposition is included as a standard feature in many available scientific computing software packages.

The matrix (I $\left.-\mathbf{A}^{+} \mathbf{A}\right)$ corresponds to the orthogonal projector on the null space of the operator $\mathbf{A}$. This means that for any arbitrary vector $\mathbf{y}$, the vector $\left(\mathbf{I}-\mathbf{A}^{+} \mathbf{A}\right) \mathbf{y}$ belongs to the null space of $\mathbf{A}$, so that $\mathbf{A}\left(\mathbf{I}-\mathbf{A}^{+} \mathbf{A}\right) \mathbf{y}=0$, a result which follows directly from Eqs. (24). Note also that Eq. (22) is a particular case of Eq. (23), with $\mathbf{A}^{+}$ $=\left(\mathbf{A}^{\mathrm{T}} \mathbf{A}\right)^{-1} \mathbf{A}^{\mathrm{T}}$.

Different choices of $\mathbf{y}$ lead to different values of $\mathbf{c}$ in Eq. (23), and hence to different correcting elements. All of them will give rise to the same minimum value for $\| \mathbf{A c}$ - $\mathbf{d} \|$, and thus all correspond to the same minimum value for $\eta$. Among these infinite solutions, there are two that are particularly interesting. One of them is obtained setting $\mathbf{y}=0$ : In this case we obtain a minimumnorm solution for c, which will be denoted henceforth by $\mathbf{c}^{\mathbf{0}}=\mathbf{A}^{+} \mathbf{d}$. This solution corresponds to the correcting element of smaller volume, which is an advantageous condition from the manufacturing viewpoint.

However, the minimum-norm solution for c will not generally provide the best optical quality. Note that all solutions of Eq. (23) are equivalent in terms of field uniformity, but they do not give the same variance. Stated otherwise, $\sigma^{2}(\boldsymbol{\alpha})$ will be uniform across the field but some solutions for $\mathbf{c}$ will give smaller values for $\sigma^{2}(\boldsymbol{\alpha})$ than others. Since the vector $\mathbf{y}$ can be chosen freely we can look for the one that minimizes the average variance:

$$
\left\langle\sigma^{2}(\boldsymbol{\alpha})\right\rangle=\frac{1}{M} \sum_{k=1}^{M} \sigma^{2}\left(\boldsymbol{\alpha}_{k}\right)=\frac{1}{M} \sum_{k=1}^{M} \sum_{i=1}^{N}\left[a_{i}\left(\boldsymbol{\alpha}_{k}\right)+c_{i}\right]^{2} .
$$

Rewriting Eq. (23) as $\mathbf{c}=\mathbf{c}^{\mathbf{0}}+\mathbf{P y}$ with $\mathbf{P}=(\mathbf{I}$ $-\mathbf{A}^{+} \mathbf{A}$ ) and substituting for the components of $\mathbf{c}$ in Eq. (25) we get

$$
\left\langle\sigma^{2}(\boldsymbol{\alpha})\right\rangle=\frac{1}{M} \sum_{k=1}^{M} \sum_{i=1}^{N}\left[a_{i}\left(\boldsymbol{\alpha}_{k}\right)+c_{i}^{0}+\sum_{j=1}^{N} P_{i j} y_{j}\right]^{2} .
$$

Setting to zero the partial derivatives of $\left\langle\sigma^{2}(\boldsymbol{\alpha})\right\rangle$ with respect to the $y_{j}(j=1, \ldots, N)$, and taking into account that $\mathbf{P}^{\mathrm{T}}=\mathbf{P}, \mathbf{P P}=\mathbf{P}$, and $\mathbf{P c}^{\mathbf{0}}=0$, we finally get

$$
\mathbf{c}=\mathbf{c}^{\mathbf{0}}+\mathbf{P}\langle\mathbf{a}\rangle=\mathbf{A}^{+} \mathbf{d}+\left(\mathbf{I}-\mathbf{A}^{+} \mathbf{A}\right)\langle\mathbf{a}\rangle,
$$

where $\langle\mathbf{a}\rangle$ is an $N \times 1$ vector whose components are the field averages of the eye aberration corresponding to each Zernike mode $\left\langle a_{i}(\boldsymbol{\alpha})\right\rangle$ given by

$$
\left\langle a_{i}(\boldsymbol{\alpha})\right\rangle=\frac{1}{M} \sum_{k=1}^{M} a_{i}\left(\boldsymbol{\alpha}_{k}\right) .
$$

The solution given by Eq. (27) corresponds to the vector c of modal coefficients for the optimal compensating element which gives the smallest uncompensated residual aberration among all those providing the best level of uniformity (minimum $\eta$ ), and hence the best possible optical quality compatible with uniformity.

\section{EXPECTED IMAGE QUALITY FOR WIDE- FIELD-COMPENSATED EYES}

The results of Section 3 will here be applied to the design and performance evaluation of wide-field compensating elements by using the data of Fig. 1. Figure 4 shows wrapped plots of the wave aberration for subject EM, with the gray scale levels spanning one wavelength of optical path $(543 \mathrm{~nm})$. Each column corresponds to a different field angle as noted. All terms in the aberration function $W_{\mathrm{e}}(\mathbf{r}, \boldsymbol{\alpha})$ have been included except for the tilt. The corresponding retinal PSFs are shown in Fig. 5. The upper row in Fig. 4 corresponds to the uncompensated case. Here the wave aberration shows variable amounts of defocus, astigmatism, and high-order aberrations. The uncompensated retinal PSFs (upper row of Fig. 5) show the noticeable contribution of astigmatic and comalike components.

The second row of Figs. 4 and 5 corresponds to the residual wave aberration after compensation with a correcting element designed to cancel perfectly the on-axis optical defects, that is, choosing the weights $\omega=\left[\begin{array}{lll}1 & 0 & 0\end{array}\right]$. As expected, the on-axis residual wave aberration is zero and the corresponding PSF is a diffraction-limited Airy disk. In this case, the residual aberration at $5^{\circ}$ of eccentricity is definitely small. At $10^{\circ}$, however, the PSF is rather broad, although much smaller than the original uncompensated one.

The third row of Figs. 4 and 5 shows the effects of using a correcting element choosing $\boldsymbol{\omega}=(1 / 2)\left[\begin{array}{lll}1 & 1 & 0\end{array}\right]$. This option gives rise to a residual aberration of exactly the same 


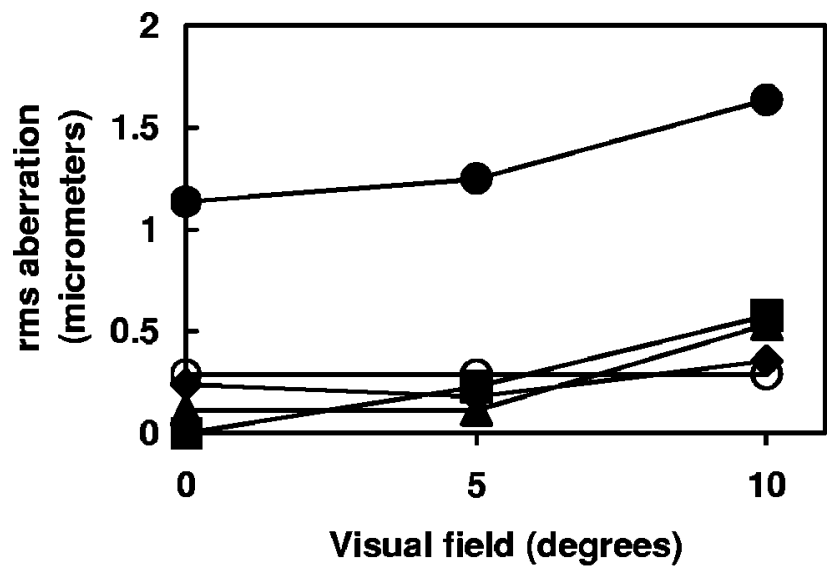

(a)

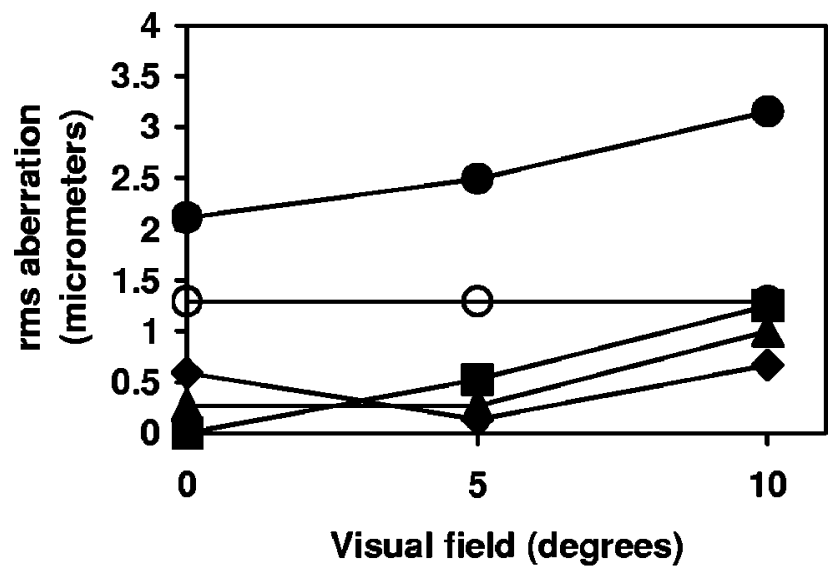

(b)

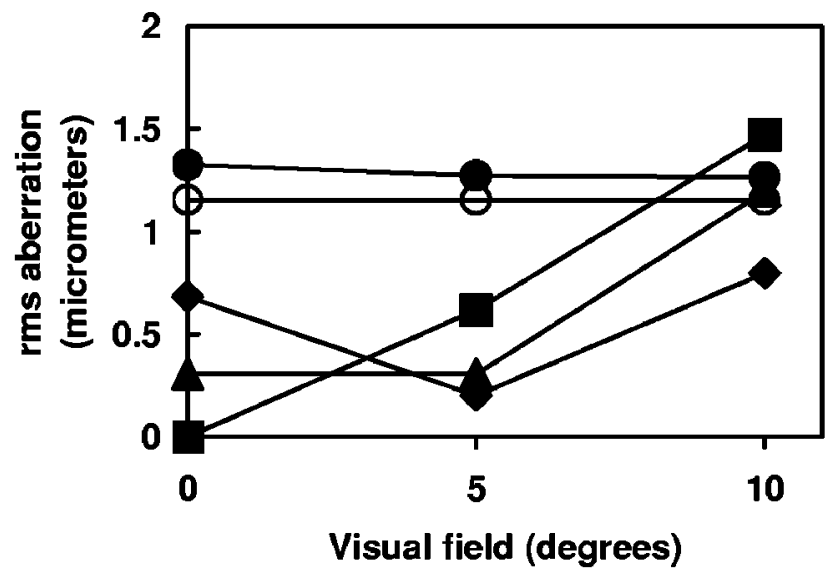

(c)

Fig. 6. Residual rms aberration for subjects (a) EM, (b) RN, and (c) CD. - before compensation; after compensation with an element designed with $\mathbf{\square}, w=\left[\begin{array}{lll}1 & 0 & 0\end{array}\right] ; \boldsymbol{\Lambda}, w=(1 / 2)\left[\begin{array}{lll}1 & 1 & 0\end{array}\right] ; \bullet$, $w=(1 / 3)\left[\begin{array}{lll}1 & 1 & 1\end{array}\right] ; \bigcirc$, after compensation with optimum coefficients for maximum uniformity for three positions across the visual field. The pupil diameter is $6.7 \mathrm{~mm}$ and the wavelength $543 \mathrm{~nm}$.

magnitude but opposite sign at the two field positions $0^{\circ}$ and $5^{\circ}$. (The corresponding gray scale aberration patterns have the same shape but reversed contrast.) Conse- quently the retinal PSF will have the same size for both locations, as can be appreciated in Fig. 5.

The fourth row of Figs. 4 and 5 corresponds to $\boldsymbol{\omega}$ $=(1 / 3)\left[\begin{array}{lll}1 & 1 & 1\end{array}\right]$. In this case the residual wave aberration tends to behave more uniformly and the size of the PSF is strongly reduced at $10^{\circ}$, at the cost of some spreading at $0^{\circ}$ and $5^{\circ}$.

Finally the fifth rows of Figs. 4 and 5 show the residual aberration and the corresponding PSFs, respectively, using a correcting element designed according to the uniform residual variance approach. Note the smaller residual aberration at $10^{\circ}$ and the slightly higher same at $0^{\circ}$ and $5^{\circ}$ in comparison with the fourth row.

Despite the expected intersubject variability, the compensation trade-offs are qualitatively similar for all three subjects here analyzed, as shown in Fig. 6. The residual rms wave aberration is plotted against field position in Figs. 6(a)-6(c) for EM, RN, and CD, respectively (only tilts are excluded from $W_{e}$ ). In each plot we show the original $\mathrm{rms}$ aberration (filled circles) and the residual ones corresponding to $\boldsymbol{\omega}=\left[\begin{array}{lll}1 & 0 & 0\end{array}\right]$ (squares), $\boldsymbol{\omega}=(1 / 2)$ $\times\left[\begin{array}{lll}1 & 1 & 0\end{array}\right]$ (triangles) and $\boldsymbol{\omega}=(1 / 3)\left[\begin{array}{lll}1 & 1 & 1\end{array}\right]$ (diamonds). The uniform rms case, achieved with a correcting element designed according to Eq. (27), is represented by open circles. The trade-offs here are roughly expressed as a balancing of the minimum rms value attainable for a given position (e.g., the on-axis one) and the resulting rms aberration range (maximum minus minimum values across the field). Note that it is possible to design an optimal correcting element yielding a minimum constant value for the residual aberration variance at the three visual field locations, although the practical relevance of this result depends strongly on the subject. While the particular aberration pattern of subject EM would allow us to design a wide-field compensating element with both small and uniform residual aberration, the aberration pattern of subject CD means that the cost of obtaining a uniform field is so high that the resulting residual aberration is close to the uncompensated case. Thus for this subject the uniform design will be almost useless. Subject $\mathrm{RN}$ represents an intermediate case for whom we could get a uniform field but at the price of losing about $50 \%$ of the compensation which could be attainable at each point with other compensation schemes.

\section{DISCUSSION}

This work addressed the problem of finding analytic solutions for the wide-field compensation of monochromatic eye aberrations. The main hypothesis used here is summarized in Eq. (5), namely, the transmittance function of the compensating element is not dependent on the angle of incidence of light. This assumption is reasonable for thin optical elements such as phase plates and deformable mirrors as long as the incidence angles are kept sufficiently small $\left(\right.$ say, $\leqslant 10^{\circ}$ ). This can be checked by exact ray-tracing through typical elements of this kind: The variation of the corresponding Zernike coefficients of the correcting elements across this field is negligibly small in comparison with that of the typical eye aberra- 
tions. An improvement of this design procedure may take into account the field dependence of the correcting element.

The work here developed uses the residual rms eye aberration as an optical quality metric. This is by no means the only possible choice, although it allows for straightforward optimization procedures and provides useful analytic results. It also provides a reasonable indication of the expected image quality as long as the residual aberrations are small. For large aberrations the relationship between rms aberration and image quality becomes more complex. Throughout this work, we performed linear minimizations of the cost functions without introducing constraints. This is a direct approach, although more elaborate ones could be useful in some situations (e.g., holding a determined aberration within some established limits). Further, we have considered cost functions which assign an equal weighting function to all Zernike modes: This restriction could be easily removed if advisable. Specific weighting of individual Zernike modes can be achieved by replacing the overall function $\omega(\boldsymbol{\alpha})$ in Eq. (9) by a set of functions $\omega_{i}(\boldsymbol{\alpha})$ introduced as factors multiplying the terms of the summation in Eq. (8). In addition, we have restricted our approach to the use of a single compensating element, which does not allow for true field-variant aberration compensation. More sophisticated compensation schemes could eliminate this constraint, too.

Optically thin correcting elements on flat or nearly flat substrates, such as those considered in this paper, and located in a plane conjugated to the eye's pupil, are usual in advanced systems for high-resolution retinal imaging. One may ask to what extent these results are of application for the design of other kinds of elements, e.g., contact lenses on curved substrates. This question has to be addressed through numerical ray tracing and has not been considered here. Nevertheless the modal coefficients given by Eqs. (16) and (27) should provide a reasonable set of initial conditions for the nonlinear optimization routines necessary to solve this design.

As a final remark, the optimization procedures developed in this paper can be applied to different problems in the design of eye aberration compensation. The parameter $\boldsymbol{\alpha}$, here a two-dimensional field position vector, may in general represent any variable or set of variables on which the eye aberration coefficients may depend (accommodation, wavelength, etc.). Within the range of validity of Eq. (5), the results here developed can be directly applied to a wide range of situations, e.g., to find the optimum coefficients for compensating elements giving the best optical quality for a wide range of accomodative states.

\section{ACKNOWLEDGMENTS}

This work has been supported by the Spanish Ministerio de Ciencia y Tecnología, Plan Nacional de Investigación Científica, Desarrollo e Innovación Tecnológica (I + D + I), DPI2002-04370-C02.

Corresponding author Salvador Bará can be reached by e-mail atbara@usc.es.

\section{REFERENCES}

1. H. von Helmholtz, Popular Scientific Lectures, M. Kline, ed. (Dover, New York, 1962).

2. M. Born and E. Wolf, Principles of Optics (Pergamon, Oxford, UK, 1987), Chap. 5, pp. 203-207.

3. M. Born and E. Wolf, Principles of Optics (Pergamon, Oxford, UK, 1987), pp. 464-468, 767-772.

4. J. Y. Wang and D. E. Silva, "Wave-front interpretation with Zernike polynomials,” Appl. Opt. 19, 1510-1518 (1980).

5. D. Malacara and S. L. DeVore, "Interferogram evaluation and wavefront fitting," in Optical Shop Testing, D. Malacara, ed. (Wiley, New York, 1992), Chap. 13, pp. 455-499.

6. L. N. Thibos, R. A. Applegate, J. T. Schwiegerling, R. Webb, and VSIA Standards Taskforce members, "Standards for Reporting the Optical Aberrations of Eyes," in Vision Science and Its Applications, V. Lakshminarayanan, ed., Vol. 35 of OSA Trends in Optics and Photonics Series (Optical Society of America, Washington, D.C., 2000), pp. 232-244.

7. H. C. Howland and B. Howland, "A subjective method for the measurement of the monochromatic aberrations of the eye," J. Opt. Soc. Am. 67, 1508-1518 (1977).

8. J. Liang, B. Grimm, S. Goelz, and J. Bille, "Objective measurement of wave aberrations of the human eye with the use of a Hartmann-Shack wave-front sensor," J. Opt. Soc. Am. A 11, 1949-1957 (1994).

9. J. Liang and D. R. Williams, "Aberrations and retinal image quality of the normal human eye," J. Opt. Soc. Am. A 14, 2873-2883 (1997).

10. P. M. Prieto, F. Vargas-Martin, S. Goelz, and P. Artal, "Analysis of the performance of the Hartmann-Shack sensor in the human eye," J. Opt. Soc. Am. A 17, 1388-1398 (2000).

11. R. Navarro and E. Moreno-Barriuso, "Laser ray-tracing method for optical testing," Opt. Lett. 24, 951-953 (1999).

12. E. Moreno-Barriuso and R. Navarro, "Laser ray-tracing versus Hartmann-Shack sensor for measuring optical aberrations in the human eye," J. Opt. Soc. Am. A 17, 974-985 (2000).

13. E. Moreno-Barriuso, S. Marcos, R. Navarro, and S. A. Burns, "Comparing laser ray tracing, spatially resolved refractometer and Hartmann-Shack sensor to measure the ocular wavefront aberration," Optom. Vision Sci. 78, 152156 (2001).

14. R. H. Webb, C. M. Penney, and K. P. Thompson, "Measurement of ocular wavefront distortion with a spatially resolved refractometer," Appl. Opt. 31, 3678-3686 (1992).

15. J. C. He, S. Marcos, R. H. Webb, and S. A. Burns, "Measurement of the wave-front aberration of the eye by a fast psychophysical procedure," J. Opt. Soc. Am. A 15, 2449-2456 (1998).

16. J. Liang, D. R. Williams, and D. T. Miller, "Supernormal vision and high-resolution retinal imaging through adaptive optics,” J. Opt. Soc. Am. A 14, 2884-2892 (1997).

17. L. Zhu, P. Sun, D. W. Bartsch, W. R. Freeman, and Y. Fainman, "Adaptive control of a micromachined continuousmembrane deformable mirror for aberration compensation," Appl. Opt. 38, 168-176 (1999).

18. E. J. Fernández, I. Iglesias, and P. Artal, "Closed-loop adaptive optics in the human eye," Opt. Lett. 26, 746-748 (2001).

19. G. D. Love, "Wave-front correction and production of Zernike modes with a liquid-crystal spatial light modulator," Appl. Opt. 36, 1517-1524 (1997).

20. F. Vargas-Martin, P. M. Prieto, and P. Artal, "Correction of the aberrations in the human eye with a liquid-crystal spatial light modulator: limits to performance," J. Opt. Soc. Am. A 15, 2552-2562 (1998).

21. R. Navarro, E. Moreno-Barriuso, S. Bará, and T. Mancebo, "Phase plates for wave-aberration compensation in the human eye," Opt. Lett. 25, 236-238 (2000).

22. S. A. Burns, S. Marcos, A. E. Elsner, and S. Bará, "Contrast improvement for confocal retinal imaging using phase correcting plates," Opt. Lett. 27, 400-402 (2002).

23. R. Navarro, E. Moreno, and C. Dorronsoro, "Monochromatic aberrations and point-spread functions of the human eye 
across the visual field," J. Opt. Soc. Am. A 15, 2522-2529 (1998).

24. L. Zhu, D. W. Bartsch, W. R. Freeman, P. Sun, and Y. Fainman, "Modeling human eye aberrations and their compensation for high-resolution retinal imaging," Optom. Vision Sci. 75, 827-839 (1998)

25. G. Smith, D. A. Atchinson, C. Avudainayagam, and K. Avudainayagam, "Designing lenses to correct peripheral refractive errors of the eye," J. Opt. Soc. Am. A 19, 10-18 (2002).

26. A. Guirao, J. Porter, D. R. Williams, and I. G. Cox, "Calculated impact of high-order monochromatic aberrations on retinal image quality in a population of human eyes," J. Opt. Soc. Am. A 19, 620-628 (2002).

27. G. Y. Yoon and D. R. Williams, "Visual performance after correcting the monochromatic and chromatic aberrations of the eye," J. Opt. Soc. Am. A 19, 266-275 (2002).

28. S. Bará, T. Mancebo, and E. Moreno-Barriuso, "Positioning tolerances for phase plates compensating aberrations of the human eye," Appl. Opt. 39, 3413-3420 (2000).

29. J. Porter, A. Guirao, I. G. Cox, and D. R. Williams, "Monochromatic aberrations of the human eye in a large population," J. Opt. Soc. Am. A 18, 1793-1803 (2001).

30. J. Herrmann, "Least-squares wave front errors of minimum norm,” J. Opt. Soc. Am. 70, 28-35 (1977).

31. B. P. Medoff, "Image reconstruction from limited data: theory and applications in computerized tomography," in Image Recovery: Theory and Application, H. Stark, ed. (Academic, New York, 1987), Chap. 9. 